\title{
MODELING ION EXTRACTION FROM AN ECR ION SOURCE*
}

\author{
B. P. Cluggish ${ }^{\#}$, S. A. Galkin, J. S. Kim, FAR-TECH, Inc., San Diego, CA 92121, U.S.A.
}

\section{Abstract}

Electron cyclotron resonance ion sources (ECRIS) that generate multiply charged ions reduce the cost to produce radioactive ion beams by reducing the accelerating voltage needed to achieve the desired beam energy. FAR$\mathrm{TECH}$, Inc. is developing an integrated suite of numerical codes to simulate ECRIS ion capture, charge breeding, and ion extraction. Ion extraction is modeled with a particle in cell (PIC) type code. Since the ion dynamics are strongly dependent on the shape of the narrow plasma sheath (plasma meniscus) at the boundary between the ECRIS plasma and the ion optics, the PIC code uses an adaptive Poisson solver to accurately resolve the sheath.

\section{INTRODUCTION}

Positive ions are extracted from the plasma in an ECRIS [1] by biasing the device at a high potential with respect to a grounded puller electrode. The large electric fields in the extraction region keep the plasma electrons confined in the ECRIS while accelerating the ions to energies large compared to their thermal energies. An innovative particle in cell (PIC) [2] type code, named IonEx [3] has been developed to simulate the ion extraction process.

At present, IonEx is a two-dimensional (2D), electrostatic code that calculates ion trajectories in cylindrical geometry under the influence of both the vacuum and self-consistent space-charge electric fields. The ion space charge is calculated from the ion trajectories by distributing the ion charge onto the computational nodes after each time step. The plasma electrons are modeled by assuming a Boltzmann distribution for the electron space charge:

$$
n_{e}=n_{0} \exp \left(\frac{e\left(\phi_{0}-\phi\right)}{T_{e}}\right) \text {. }
$$

Here $n_{e}$ is the electron density, $e$ is the electron charge, $\phi$ is the potential, $T_{e}$ is the electron temperature, and $\phi_{0}$ and $n_{0}$ are the potential and density at prescribed at a boundary.

The application of the extraction voltage leads to the formation of a narrow plasma sheath, or "plasma meniscus," in the extraction aperture. For typical ECRIS parameters this meniscus may be a centimeter in diameter but only microns thick. Accurately modeling ion extraction requires properly resolving the shape of the meniscus. In order to do this with a reasonable number of computational nodes (points), a particle in cloud of points technique (PICOP) [3] has been implemented.

While similar to the often used adaptive mesh

\footnotetext{
*Work supported by US DOE

"cluggish@far-tech.com
}

refinement (AMR) technique, the meshfree technique used in IonEx should be more flexible, because only the locations of the computational nodes are used in the calculation and not the connections between the points. This flexibility should be especially advantageous when the code is extended to three-dimensions (3D). While other PIC codes have been developed to model the extraction of ions from a plasma [4, 5], to our knowledge IonEx is the only PICOP code that does so.

\section{PICOP VS. PIC}

The PICOP technique is discussed in detail in [3]. It differs from the PIC technique in that PICOP uses an unstructured cloud of points instead of a rectangular or even an unstructured mesh. This difference affects 3 aspects of the operation of the code:

1. Distribution of the particle charge to the computational nodes, and calculation of the electric field at the location of the particle

2. Solution of Poisson's equation

3. Dynamic adaptation of the computational nodes to the solution structure during the solution process

To distribute the charge of a particle to the grid points in a PIC code, the cell in which the particle resides must first be determined. The charge on the particle is then distributed among the points at the vertices of the cells. The reverse procedure is used to find the electric field at the location of the particle; the electric field is given by a weighted average of the electric field at the vertices of the cell. In a PICOP code, on the other hand, there are no cells. Instead, the charge of a particle is distributed to all of the points within a certain radius of the particle, and electric field at the location of the particle is given by a weighted average of the electric field at those points. The algorithm is similar to that used in cloud-in-cell codes.

The cell geometry also defines how Poisson's equation is solved in a PIC code. Mesh based Poisson solvers use finite element, finite volume, finite difference, and other mesh-based methods to solve the field equation(s). Once written as a program, is difficult or impossible to change the form of the equation or the order to which it is approximated without essentially altering the code. In contrast the meshfree technique used in PICOP is flexible enough that both the type of equation and the order of the approximation can be changed if needed without any essential modification of the code.

The current node/point adaptation algorithm used in IonEx is based on the local refinement grid adaptation technique [6]. The point generator analyzes an error indicator, which is based on the gradient (the first order derivatives) and the Hessian matrix of the second order 
derivatives of a monitor function. The monitor function provides information to the point generator as to how and where to refine or coarsen the grid. The monitor function is a weighted sum of different functions (such as ion density, electron density, and potential), which represent the solution to the entire problem.

\section{TYPICAL IONEX RESULTS}

We have used the IonEx code to simulate the extraction of singly charged hydrogen ions from an ECRIS with the parameters shown in Table 1.

Table 1: Parameters for IonEx simulations

\begin{tabular}{|l|l|l|l|l|}
\hline$n_{0}\left(\mathrm{~m}^{-3}\right)$ & $T_{e}(\mathrm{eV})$ & $U_{i}(\mathrm{eV})$ & $\Phi_{1}(\mathrm{~V})$ & $\Phi_{2}(\mathrm{~V})$ \\
\hline $2.24 \times 10^{17}$ & 20 & 20 & 65000 & 0 \\
\hline
\end{tabular}

Here, $U_{i}$ is the initial ion energy, and $\Phi_{1}$ and $\Phi_{2}$ are the potentials of the two extraction electrodes. The geometry of the extraction region is shown in Figure 1. The plasma is on the left hand side; the plasma potential $\phi_{0}$ and the plasma density $n_{0}$ are assumed to be uniform along the left hand boundary. There are two electrodes. Electrode 1 is the wall of the plasma chamber where the extraction aperture is located; it is biased to a high positive voltage. We assume that the plasma potential differs from the voltage on electrode 1 by the standard sheath potential $\phi_{s}$ :

$$
\phi_{0}-\Phi_{1} \equiv \phi_{s}=\frac{T_{e}}{2} \ln \left(\frac{m_{i}}{\pi m_{e}} \frac{T_{e}}{U_{i}}\right)
$$

where $m_{e}$ and $m_{i}$ are the electron and ion masses, respectively. Electrode 2 is grounded to extract the ions and repel the plasma electrons. The system is cylindrically symmetric, with constant potential (Dirichlet) boundary conditions on the left hand and electrode boundaries; and zero perpendicular electric field (Neumann) boundary conditions on the other boundaries.

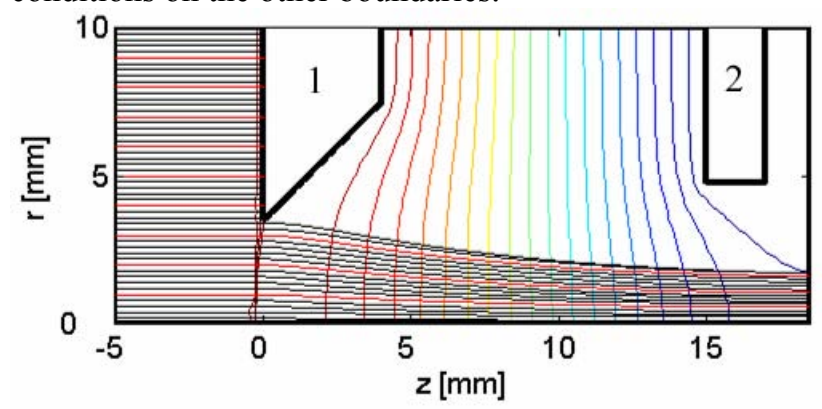

Figure 1: Electrodes, ion trajectories (black), and equipotential lines (multi-colored contours) calculated by IonEx using the parameters in Table 1. Only 50 trajectories are shown; some are drawn in red for easier visualization.

The ion trajectories and potential contours are plotted in Figure 1. The ions enter from the left with kinetic energy $U_{i}$, and the ion current density, $j_{i}$, is assumed to be

$$
j_{i}=n_{0} \sqrt{\frac{2 U_{i}}{m_{i}}} .
$$

The ion current is divided among 500 test particles. The ion temperature is assumed to be zero and their velocity is assumed to be purely axial. The ions first pass through the plasma, where the electric field is weak, until they pass through the sheath. The ions then either hit electrode 1 and are removed from the system, or are extracted and accelerated by the potential difference between the plasma and electrode 2 .

The trajectories are initially straight because the electric field is weak in the plasma. The equipotential contours from the tip of electrode 1 to the axis at $z=0$ show the location of the plasma meniscus. Here the ions begin to accelerate as the potential falls. The ions are then further accelerated and the beam narrows due to the electric fields between electrodes 1 and 2 .

Figure 2 shows a detail of the equipotential contours in the plasma meniscus around the tip of electrode 1 . The potential drops by $3 T_{e}$ in an axial distance of less than 0.2 $\mathrm{mm}$. The electron density drops by a factor of 20 in the same distance. This steep gradient necessitates a high density of computational nodes to be accurately modeled.

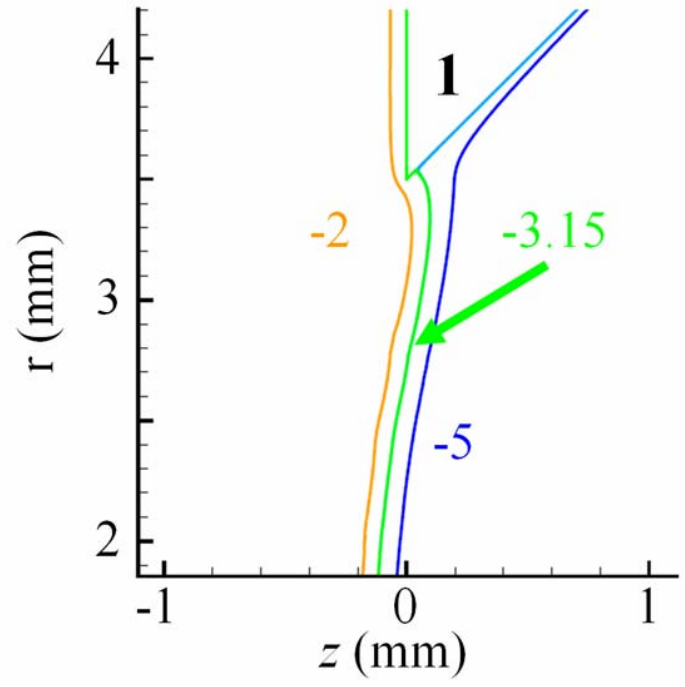

Figure 2: Contours of $e\left(\phi-\phi_{0}\right) / T_{e}$ in the plasma meniscus around the tip of electrode 1 .

The locations of the computational nodes for this solution are shown in Figure 3.

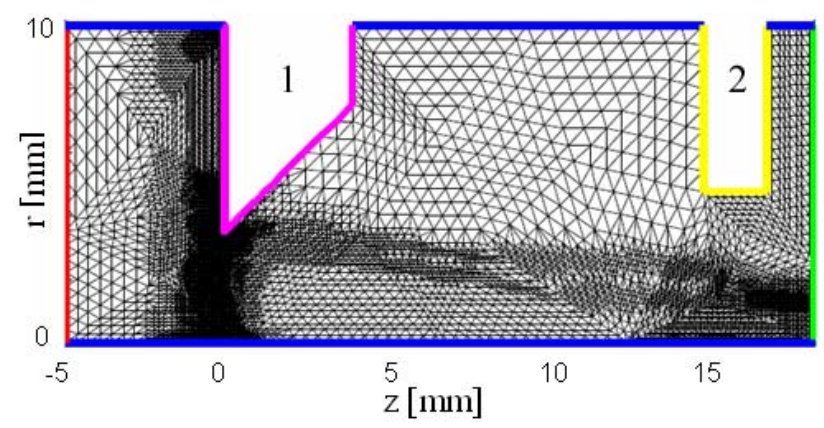

Figure 3: Locations of computational nodes/points. Different colors on the boundaries signify different boundary conditions. Lines connecting the nodes are for visualization only, and are not used in the calculation. 
The final number of points was about 15,000, with inter-point spacing as small as $5 \mu \mathrm{m}$. Note that IonEx puts a high density of nodes around the plasma sheath, the sharp corner of electrode 1 , and the edge of the ion beam. Regions with low density and/or electric fields, such as the vacuum region between the two electrodes, are assigned only a low density of nodes.

\section{COMPARISON WITH IGUN}

We have benchmarked the IonEx code against the widely used IGUN PIC code [4]. We find that the trajectories and potential profiles are in good agreement with the IonEx results. IGUN was run in a constant plasma density mode, using a rectangular grid with 94000 nodes, compared to 15000 nodes for IonEx.

We have also compared the emittance of the extracted ion beam as calculated by the two codes. Figure 4 shows an emittance plot (trajectory angle vs. transverse position) for the axial location of the right hand boundary, as calculated by each code. All of the trajectories that intersect the right hand boundary are included in the emittance plot. The two codes are in good agreement, with the notable exception that a few of the particles at the edge of the beam in the IonEx run have steeper trajectories (larger angle with respect to the axis) than their counterparts in IGUN.

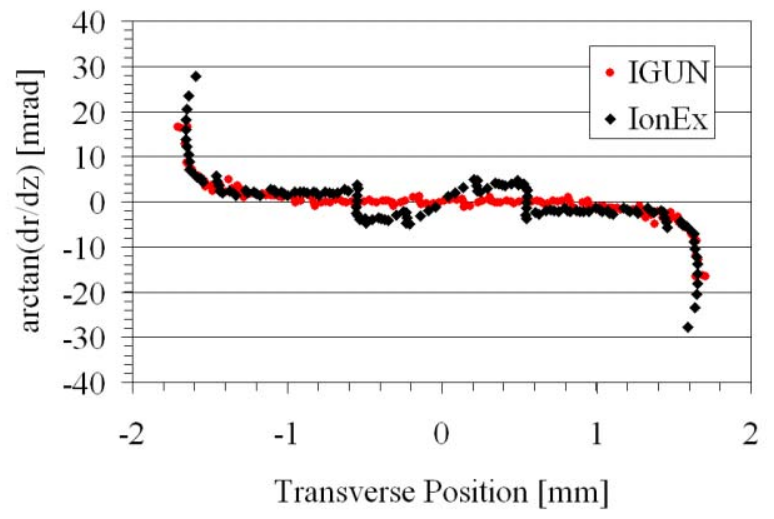

Figure 4: Comparison of the emittance of the extracted beam as calculated by between IonEx and IGUN for the parameters in Table 1. Axial location is at the right hand boundary.

These are particles that pass very close to the sharp tip of electrode 1, and are influenced by the strong electric fields in that region. The discrepancy between IGUN and IonEx can probably be explained by the way each code calculates the electric fields in this region. IGUN uses a 1D analytic sheath model to calculate potentials above that on electrode 1 (i.e. $\phi>\Phi_{1}$ ), and ignores the Boltzmann electron term in Poisson's equation where the potential is less than that on electrode 1. This 1D approach may not be accurate in the vicinity of the electrode tip, which has a small radius of curvature. In contrast, IonEx calculates the electric field in 2D, including the Boltzmann electron term, over the whole domain; and extra computational nodes are added to correctly model the electric field near the electrode tip, as shown in Figure 3. We plan to continue to investigate the discrepancies between the two codes.

Finally, we have studied the effect of multiple charge states on the beam emittance. Using the same parameters as in Table 1, we simulated the simultaneous extraction of singly and doubly charged helium ions, $\mathrm{He}+1$ and $\mathrm{He}+2$. We assumed that the both species had the same initial energy, due to collisional coupling in the plasma, and that the ratio of +1 to +2 ions was $7 / 3$. An emittance plot for both charge states is shown in Figure 5. We found that the trajectories are nearly independent of the charge state, and both species have nearly the same emittance.

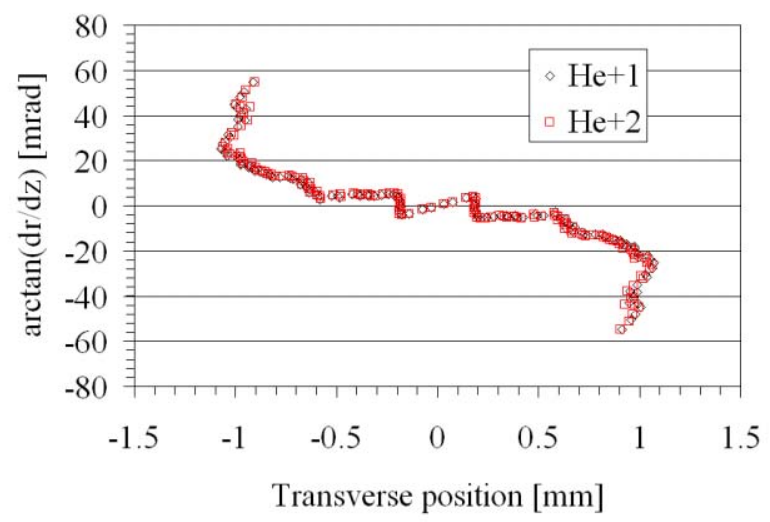

Figure 5: Emittance plot for $\mathrm{He}+1$ and $\mathrm{He}+2$ ions.

The reason the trajectories are nearly independent of the charge state is that the initial energy of the ions is small compared to their final energy and the axial velocity is large compared to the radial velocity. This means that to first order both species travel in straight lines, the only difference in their trajectories being that the +2 ions move faster than the +1 ions.

\section{REFERENCES}

[1] R. Geller, Electron Cyclotron Resonance Ion Sources and ECR Plasmas, Institute of Physics Publishing, Bristol, Philadelphia, (1996)

[2] C. K. Birdsall and A. B. Langdon, Plasma Physics via Computer Simulation, (1985) McGraw-Hill

[3] S. A. Galkin, B. P. Cluggish, J. S. Kim, and S. Y. Medvedev, "Advanced PIC Algorithm with Adaptive Meshless Field Solver," to be published in Proc. Pulsed Power and Plasma Science Conference 2007. Presented in Section 8B6, Albuquerque 17-22 June, 2007

[4] R. Becker and W.B. Hermannsfeldt, "IGUN-A program for the simulation of positive ion extraction including magnetic fields," Rev. Sci. Instrum., 63 (4), 2756 (1992)

[5] A. Friedman et al., Part. Accel. 37-38, 131 (1992)

[6] A.A. Martynov, S.Yu. Medvedev, A Robust Method of Anisotropic Grid Generation, Workshop: "Grid generation: Theory and Applications", Moscow 2002, http://www.ccas.ru/gridgen/ggta02/abstracts.html 